\title{
NACHHALTIGKEIT ALS IMPERATIV DES ALLTAGS
}

\begin{abstract}
Seit weit mehr als einem Jahrzehnt sind wir bereits auf dem Weg zu einer nachhaltigen Entwicklung. Das ist die Folge einer geistigen Ernüchterung, die Anfang der 1980er Jahre zustande kam und aus der Einsicht erwuchs, dass es so nicht weitergehen kann. Seit jener Zeit geht die Suche nach entsprechenden Handlungs- und Verhaltensregeln voran, die das Schrumpfen von natürlichen und menschlich-sozialen Grundlagen der Wirtschaftsentwicklung verhindern. Der im Folgenden vorgeschlagene Imperativ knüpft an diese Strömung und an die Mitverantwortung für die weitere Entwicklung an. Das Konzept der nachhaltigen Entwicklung setzt auf das Auferlegen entsprechender Bedingungen. Dies betrifft auch die Kultur des Alltags. Die sich kumulierenden Auswirkungen vieler individueller Handlungen auf die Umwelt erzeugen einen sich verstärkenden Effekt, der sich als Resultat aus der Multiplikation und Aggregation individueller Entscheidungen ergibt. In den Umweltwissenschaften wird das als „Destruktion durch geringfügige Veränderungen“ oder „Tyrannei kleiner Entscheidungen“ bezeichnet. Die axiomatische Aussage „Deine alltägliche, einzelne, individuelle Entscheidung nimmt Einfluss auf die Umwelt" stellt die Grundlage dar, die den Imperativ einer Alltagsnachhaltigkeit bedingt. Zum Kern dieses Imperativs gehören u.a. die folgenden Maximen: „Sei ethisch und moralisch in deinem alltäglichen Verhalten und Handeln; überprüfe und beurteile deine alltäglichen Gewohnheiten periodisch auf Nachhaltigkeit hin und ändere sie, wenn sie sich als nicht nachhaltig erweisen; vermehre dein Wissenskapital und nutze dazu einschlägige Ratgeber, die im Hinblick auf Nachhaltigkeit richtiges Handeln definieren; sei effizient und sparsam im alltäglichen Umgang mit Umweltgütern (Wasser, Energie, Nahrung, Raum etc.); nutze Mehrzweckprodukte, Mehrwegflaschen, -einkaufstaschen und sonstige Mehrwegverpackungen statt Mehrfachverpackungen." Ohne umfangreiche, allgemeine Verbreitung und Umsetzung des Imperativs einer Alltagsnachhaltigkeit wird eine Annäherung an den Kern und das Wesen einer nachhaltigen Entwicklung nicht möglich
\end{abstract} sein.

Schlüsselbegriffe: Nachhaltigkeit, Alltagsleben, nachhaltige Entscheidungen.

\section{EINFÜHRUNG}

Die Alltagskultur ist selbstverständlich ein fester Bestandteil einer solchen Kultur, deren axiales Element der Kern der nachhaltigen Entwicklung (sustainable development) sein soll ${ }^{2}$. Vor dem Hintergrund von Nachhaltigkeit kann eine konkrete Alltagskultur

\footnotetext{
${ }^{1}$ Prof. Ord. Dr. habil. Ing. Ryszard Janikowski, Wydział Zarządzania, Górnośląska Wyższa Szkoła Handlowa [Fakultät für Management, Oberschlesische Handelshochschule], Katowice, e-mail: ryszard.janikowski@gwsh.pl

${ }^{2}$ Vgl. R. Janikowski, Kultura osia zrównoważonego rozwoju [Kultur als Achse der Nachhaltigkeit] In: R. Janikowski, K. Krzysztofek (Hrsg.), Kultura a zrównoważony rozwój. Środowisko, ład przestrzenny, dziedzictwo [Kultur und nachhaltige Entwicklung. Umwelt, Raumordnung, Erbe], Polski Komitet do spraw UNESCO [Polnisches UNESCO-Komitee], Warszawa 2009 (poln.).
} 
unterschiedlich beurteilt werden. Sowohl eine vollständig positive Bewertung, was mit der Realisierung bestimmter Nachhaltigkeitsziele zusammenhängt, als auch eine negative Bewertung, was mit Aktivitäten und Handlungen zusammenhängt, die grundsätzlich im Gegensatz zu diesen Zielen stehen, sind möglich. Der Begriff „Kultur“ hat eine multidimensionale Bedeutung ${ }^{3}$. Im Folgenden wird auf die sehr wichtige Perspektive des Alltagslebens Bezug genommen, zu dem etwa Gartengießen gehört. Diese Herangehensweise knüpft an die sich rasch entwickelnde Strömung der Alltagsforschung $\mathrm{an}^{4}$. $\mathrm{Zu}$ dieser Strömung gehören neuere Arbeiten aus dem Bereich der Soziologie des Alltags, der Anthropologie des Alltags sowie der kulturwissenschaftlichen Alltagsforschung. Soziologische Untersuchungen verweisen auf einen reflexionslosen, beinahe automatischen Ablauf des Alltäglichen. Gleichzeitig machen Populationsforschung, Sozialforschung und Volkswirtschaftslehre darauf aufmerksam, dass diese automatischen, gewohnheitsmäßigen Handlungs- und Verhaltensmuster eines einzelnen Menschen, die auf der Ebene des individuellen Lebens zu harmlosen und irrelevanten Ressourcenveränderungen führen, gravierende Auswirkungen auf die Umwelt im globalen Ausmaß herbeiführen können und häufig auch herbeiführen. Zu diesen Gefahren für die Umwelt gehören auf der Seite des Einzelnen verschwenderisches Umgehen mit Umweltressourcen, ihr übermäßiger Verbrauch und eine geringe Effizienz ihrer Nutzung.

Der in dem vorliegenden Beitrag vorgeschlagene Imperativ einer Alltagsnachhaltigkeit ist ein Versuch, die Herausforderungen anzugehen und das Alltagsleben des Einzelnen so zu gestalten, dass es dem gesellschaftlichen, politischorganisatorischen Rahmen einer nachhaltigen Entwicklung besser entspricht und diesen angemessen ergänzt. Es erfordert allerdings, dass die grundlegenden Handlungs- und Verhaltensregeln von allen Mitgliedern aller Gemeinschaften, die im Wohlstand leben wollen, wahrgenommen und gelebt werden.

\section{TYRANNEI DER ALLTÄGLICHEN ENTSCHEIDUNGEN}

Es sei mit einem Zitat eines Dialogs aus dem Internet begonnen.

Frage: Seit einiger Zeit quält mich das alltägliche „Stecker ein, Stecker aus “ beim Gebrauch von Elektrogeräten. Ich frage mich oft, wie viel Strom nachts- und tagsüber alle meine ungebrauchten Elektrogeräte wie Mikrowelle, Fernseher, Decoder, Stereoanlage etc., die im Stand-by-Modus eingeschaltet bleiben, in der Tat verbrachen? Lohnt es sich überhaupt, bei diesem ganzen Zeug täglich den Stecker zu ziehen?

Antwort: Quatsch! Du übertreibst! Dadurch, dass eine LED über die Nacht nicht brennt, wird die Welt nicht geheilt! 5 .

Im Gegensatz zur Behauptung in der Antwort soll ausdrücklich betont werden: Gerade dieses individuelle Engagement wird die Welt vor Untergang retten! In der ersten Dekade des 21. Jahrhunderts verbrauchen alle sich in ständiger Nutzungsbereitschaft (stand-by)

${ }^{3}$ Vgl. L.E. Harrison, S.P. Huntington (Hrsg.), Kultura ma znaczenie [Kultur hat Bedeutung] Wydawnictwo Zysk i S-ka, Poznań 2003 (poln.); R. Janikowski, Kultura osią zrównoważonego rozwoju [Kultur als Achse der Nachhaltigkeit], op. cit.

${ }^{4}$ L. Head, P. Muir, Changing cultures of water in eastern Australian backyard gardens. In: Social \& Cultural Geography, 8 (6) 2007, S. 889-905.

${ }^{5}$ Siehe hierzu: http://www.elektroda.pl/rtvforum/topic1400058.html [Update: 3/09/2010]. 
befindenden elektrischen Haushaltsgeräte in den hochentwickelten Ländern etwa $10 \%$ der gesamten elektrischen Nutzungsenergie ${ }^{6}$.

Wenn man dabei die Perspektive der Erschöpfbarkeit von Ressourcen, darunter insbesondere von fossilen Energieträgern, einbezieht, kann man das gleiche, nun aber modifizierter sagen: Die sich in ständiger Nutzungsbereitschaft befindenden Haushaltsgeräte in den Industrieländern verschwenden ca. $10 \%$ der weltweit verwendeten elektrischen Energie. Anfang des 21. Jahrhunderts trifft das auf eine ganze Reihe von Geräten wie Fernseher, Funkgeräte, CD- und DVD-Player, Computer, Drucker, Overhead-Projektor, Kaffeemaschinen und viele andere zu. Andererseits ist dieser Energieaufwand aus der Sicht unserer menschlichen Bequemlichkeit ein Äquivalent menschlicher Arbeit, die für das Ein- und Ausschalten dieser Geräte sowie für das Herangehen und sich Entfernen von diesen Geräten selbst verwendet wird. Viele andere Beispiele für einen Übergang der Arbeit des Menschen in die Arbeit der Energie, der als einer der Grundzüge unseres Zivilisationsprozesses anerkannt wird, finden sich in der Literatur $^{7}$. Paradigmatisch seien hier die Arbeit der Waschfrau, die durch elektrisch betriebene automatische Waschmaschine ersetzt wurde, die Arbeit des Heizers, die durch die Einführung von programmierbaren Gasheizkesseln überflüssig wurde, oder die Arbeit der Gaslampenbrenner, die durch moderne ferngesteuerte elektrische Straßenbeleuchtungssysteme ersetzt wurde, genannt. Die sich kumulierenden Folgen vieler individueller Handlungen und Entscheidungen für die Umwelt bergen wechselseitige Verstärkungspotenziale in sich, die sich aus Synergien, Multiplikationen und Aggregationen zwischen diesen individuellen Entscheidungen ergeben. In den Umweltwissenschaften wird dieses Phänomen als „Destruktion durch geringfügige Veränderungen“ oder „Tyrannei kleiner Entscheidungen“ bezeichnet ${ }^{8}$. Die Tyrannei kleiner Entscheidungen kommt überall vor und bezieht sich auf ganz einfache alltägliche Entscheidungen eines jeden Menschen, etwa Entscheidungen für oder gegen Mülltrennen, Gartengießen oder Waschen eines Strumpfs mit Hand oder mit Hilfe einer großen Waschmaschine. Dazu gehören Entscheidungen, ob man den Fernseher, den Computer oder eine Hifi-Anlage vollständig aus dem Netz ausschaltet, ob man sein Auto an einem Fluss wäscht oder lieber in eine Autowaschanlage fährt oder Entscheidungen darüber, welche Wassermengen man für das Toilettenspülen verbraucht, und viele ähnlich bedeutungslose kleine Entscheidungen.

\footnotetext{
${ }^{6}$ Vgl. A. Meier, Standby: Where are we now? In: Proceedings of ECEEE 2005 summer study: What works and who delivers? Paris 2005: European Council for Energy Efficient Economy; K. GramHanssen, Consuming technologies - developing routines. In: Journal of Cleaner Production, 16(11) 2008, S. 1181-1189; K. Gram-Hanssen, Standby Consumption in Households Analyzed With a Practice Theory Approach. In: Journal of Industrial Ecology, 14(1) 2010, S. 150-165

${ }^{7}$ Siehe hierzu: C. Ponting, A Green History of the World, Sinclair-Stevenson Ltd., London 1991.

${ }^{8}$ Vgl. D. J. Gamble, Destruction by insignificant increments. In: Northern Perspectives 7 (1979), S. 1-4; W. E. Odum, Environmental degradation and the tyranny of small decisions. In: Bioscience 32 (1982), S. 728-729; R. Janikowski, Zarzadzanie ekologiczne [Öko-Management], Akademicka Oficyna Wydawnicza PLJ, Warszawa 1999 (poln.).
} 
Jede unserer individuellen Entscheidungen, die unser Verhalten konkret determiniert, hat Einfluss auf die Quantität und Qualität der Umwelt, sowohl in positiver als auch in negativer Dimension? ${ }^{9}$.

\section{KULTUR DES ALLTAGSLEBENS}

Die Kultur unseres Alltagslebens und deren Geschichte stellt eine Geschichte des Schmutzes, des Essens, der Mode und vieler anderer (fast) täglich von uns wiederholter Handlungen/Aktivitäten dar. Sie werden alle von Kultur geprägt, dabei sind sie zugleich Ergebnisse fixierter, gefestigter Gewohnheiten und Rituale. ${ }^{10}$ „Das Essen und Trinken sind in allen ihren Aspekten gelernte, eingeübte Tätigkeiten, und ihre Form wird durch Kultur geprägt"“11 .,Auch in seinen gegenüber der Mode empfindlichsten Kontexten wird Konsum [...] durch Repetition zur Gewohnheit. Entscheidend dafür ist, dass der Konsum in allen sozialen Kontexten sich um „Techniken des Körpergebrauchs“ - wie sie von Marcel Mauss (1973) genannt werden - „konzentriert, und der Körper bestimmte Formen der Diszipliniertheit verlangt, die wiederholbar sind oder zumindest dauerhaft gelten"12. „Die Frage der Sauberkeit und Hygiene, die einerseits kulturell und biologisch bedingt, andererseits aber auch tief persönlich ist, erweckt seit jeher zahlreiche Kontroversen. Während die antiken Griechen um die Überlegenheit des warmen Bades über das kalte Bad stritten, vermieden die Europäer im 16. Jahrhundert - mit Ausnahme jener prominenten Glücksvögel, die berühmte Badeorte besuchten - jeglichen Kontakt mit Wasser, während die Bauern aus dem 19. Jahrhundert (die heute für echte Pioniere der Hygiene-Hypothese gehalten werden könnten) wiederum hartnäckig bei gesundheitsstiftenden Eigenschaften von Schmutz beharren wollten, obwohl die HygieneVerbreiter mit allen Mitteln versuchten, diese Vorstellungen und Gewohnheiten zu korrigieren" ${ }^{\text {13. }}$.

Die Alltagskultur ist nicht nur zeitlich variabel, sondern auch gesellschaftlich, sozial divers und relativ. „Die Art und Weise, wie Hygiene in einer bestimmten Kultur betrachtet und verwirklicht wird, liefert immer viele interessante Informationen über diese Kultur selbst. Die Franzosen scheinen ihren nahezu perversen nationalen Stolz aus ihrer nonchalanten Haltung zur Sauberkeit zu schöpfen. [...] Völlig anders sieht dagegen die Körperpflege bei Amerikanern aus. Das tägliche Waschen nur bis auf die Handwurzel ist für sie absolut nicht mehr denkbar, viele scheuern sich heute so obsessiv wie nie zuvor“"14.

\footnotetext{
${ }^{9}$ Vgl. M. Pennybacker, C. Vibbert, Do One Green Thing: Saving the Earth Through Simple, Everyday Choices, St. Martin's Griffin, New York 2010; R. Janikowski, Zarzadzanie ekologiczne [Öko-Management], op. cit.

${ }^{10}$ M. Toussaint-Samat, Historia naturalna i moralna jedzenia [Natur- und Moralgeschichte der Ernährung] Wydawnictwo W.A.B., Warszawa 2002 (poln.); A. Appadurai, Nowoczesność bez granic. Kulturowe wymiary globalizacji [Die Moderne ohne Grenzen. Kulturelle Dimensionen der Globalisierung] Univeritas, Kraków 2005 (poln.); K. Ashenburg, Historia brudu [ Die Geschichte des Schmutzes], Bellona S.A., Warszawa 2007 (poln.); E. Baldwin, B. Longhurst, S. McCracken, M. Ogborn, G. Smith, Wstęp do kulturoznawstwa [Einführung in die Kulturwissenschaften], Verlag Zysk i S-ka, Poznań 2007 (poln.).

${ }^{11}$ E. Baldwin et al., op. cit., S. 328.

${ }^{12}$ A. Appadurai, Nowoczesność bez granic..., op. cit., S. 102.

${ }^{13}$ K. Ashenburg, Historia brudu, op. cit., S. 246.

${ }^{14}$ Ibidem, S. 246-247.
} 


\section{REGELN EINES NACHHALTIGEN VERHALTENS IM ALLTAG}

Das Konzept nachhaltiger Entwicklung setzt auf eine Auferlegung von Bedingungen, die die bisherigen für Natur, Mensch, Gesellschaft und Wirtschaft selbst gefährlichen Entwicklungen dämpfen und einschränken sollten. Als das übergeordnete Entwicklungsziel wird eine dauerhafte Absicherung der menschlichen Bedürfnisbefriedigung angenommen. Nachhaltigkeit knüpft an das neue Paradigma in der Wissenschaft an, das auf Interdisziplinarität, Transdisziplinarität und Multidisziplinarität beruht ${ }^{15}$, denn eine auf die Dauer abgesicherte, nachhaltige Befriedigung menschlicher Bedürfnisse erfolgt nur durch eine gegenseitig abgestimmte Realisierung der sozialen, wirtschaftlichen und umweltbezogenen Ziele, also durch eine entsprechende Gesellschafts-, Wirtschafts- und Umweltentwicklung. Dabei ist ein ständiges Verweisen auf enge Zusammenhänge und Rückkopplungen zwischen diesen drei Entwicklungskategorien, die sich einander interaktiv beeinflussen und bedingen sollten, von entscheidender Bedeutung. Der nachhaltige Entwicklungsprozess wird durch einen Satz von Handlungs- und Verhaltensprinzipien bedingt, die von allen an der Entwicklung beteiligten und die Entwicklung beeinflussenden Akteuren befolgt werden müssen. Diese Prinzipien ergeben sich inhaltlich aus den Kerngedanken des Nachhaltigkeitskonzeptes und stellen drei Säulen dar, die unmittelbar von den kanonischen Elementen gegenwärtiger Kultur herkommen:

- den Werten und Normen der Moral;

- den Naturgesetzen, vor allem den Gesetzen der Physik;

- der Leistungsfähigkeit und Effizienz des Wirtschaftens;

$\mathrm{Zu}$ den grundlegenden Prinzipien der ersten Säule (Werte und Moral) gehören vor allem:

- der Anthropozentrismus-Grundsatz - alle menschlichen Wesen sind als zentrale Subjekte des Entwicklungsprozesses anzuerkennen; als primärer Bezugspunkt jeder Entscheidung gilt das Recht jedes Menschen auf ein gesundes und kreatives Leben in Harmonie mit der Natur; als höchstes Gut gilt das menschliche Leben und menschliche Gesundheit;

- die Grundsätze der Nachhaltigkeit und der intergenerationellen Gerechtigkeit die Befriedigung gegenwärtiger Bedürfnisse darf die Möglichkeiten einer angemessenen Befriedigung ähnlicher Bedürfnisse zukünftiger Generationen nicht einschränken; alle menschlichen Generationen sollen über vergleichbare Entscheidungsmöglichkeiten über ihre eigene Entwicklung verfügen;

- der Grundsatz des Weltzentrismus und der Globalität - als Bezugssubjekt jeder Beurteilung der Entwicklung gilt für die gesamte Menschheit, die Menschengattung; jeglicher Egozentrismus und Ethnozentrismus sind strikt zu vermeiden;

$15 \mathrm{~K}$. Michalski, Interdyscyplinarność - transdyscyplinarność - multidyscyplinarność nowy paradygmat $w$ nauce $i$ badaniach [Interdisziplinarität - Transdisziplinarität - Multidisziplinarität. Ein neues Paradigma in Wissenschaft und Forschung] In: Zeszyty Naukowe Politechniki Rzeszowskiej. Ekonomia $i$ Nauki Humanistyczne [Wissenschaftliche Hefte der Technischen Hochschule Rzeszow. Ökonomie und Geisteswissenschaften] Bd. 235 (2007), H. 16, S. 83-100 (poln.). 
- allgemeine Normen des Menschenrechts - jeder einzelne Mensch hat den Anspruch, sowohl als Objekt als auch als Subjekt der Entwicklung berücksichtigt $\mathrm{zu}$ werden.

$\mathrm{Zu}$ den grundlegenden Prinzipien der zweiten, der naturwissenschaftlichen Säule gehören vor allem:

- der System-Grundsatz - alles ist mit allem verbunden und hängt mehr oder weniger eng miteinander zusammen; deshalb zieht jedes Handeln Folgen unterschiedlichen Typs (primäre, sekundäre, tertiäre Folgen) in Hinblick sowohl auf die Zeitabfolge, auf kausale Zusammenhänge als auch auf die Bewertungsrelevanz (unmittelbare, indirekte, sofort eintretende, zeitlich versetzte sowie räumlich nahe/ferne Folgen) nach sich;

- der Grundsatz der Begrenztheit und Erschöpfbarkeit von Ressourcen - die Erde hat ein begrenztes Volumen, damit sind auch ihre Ressourcen nicht unendlich; manche Naturressourcen sind uneingeschränkt erneuerbar, manche sind nur eingeschränkt erneuerbar, leider sind z.Z. die endlichen Ressourcen wie fossile Brennstoffe oder Mineralrohstoffe von größter wirtschaftlicher Bedeutung, ähnlich verhält es sich bei der Aufnahmekapazität der Umwelt für die Schadstoffemissionen und bei sonstigen Umweltresistenzen gegen anthropogene Belastungen: diejenigen Kapazitäten, die für die Sicherung menschlichen Lebens und für die menschliche Gesundheit relevant sind, sind fast alle endlich;

- der Diversitäts-Grundsatz - eine biotische, individualgenetische und populationsgenetische, ökosystemische Diversität, auf der Ebene des Humanen zusätzlich noch kulturelle, soziale oder technologische Diversität, verringern die innere Störanfälligkeit jedes Entwicklungsprozesses und stärken die äußere Widerstandfähigkeit (Resilienz) gegen schädigende Einflüsse der Umgebung.

$\mathrm{Zu}$ den grundlegenden Prinzipien der dritten Säule, die sich auf Leistungsfähigkeit und Effizienz und im Allgemeinen auf gutes Wirtschaften beziehen, gehören vor allem:

- der Grundsatz der Sparsamkeit und Effizienz - mit den Schlüsselressourcen Raum, Zeit, Stoff und Energie, aber auch mit allen anderen Ressourcen muss effektiv, effizient, sparsam und nicht verschwenderisch umgegangen werden;

- der Grundsatz der Segregation, (Re-)Zirkulation, Kreislaufwirtschaft von Stoff und Energie und Systemintegration - es gilt, die Materie, die der Umwelt einmal entzogen wurde, mehrmals im Rahmen der auf Segregation und (Re-)Zirkulation basierenden integrierten, geschlossenen Wirtschaftskreisläufe zu nutzen;

- der Grundsatz der Kapitalvermehrung und Kapitalerneuerbarkeit - es gilt, alle verfügbaren Kapitalkategorien (Natur-, Kultur-, Human-, Sozial- und Sachkapital) zu vermehren und die Nutzung von erneuerbaren Kapitalformen wie bestimmte Energiequellen oder Wissenskapital zu fördern.

Die wesentlichen Wirkungsfaktoren sollen die grundlegenden Nachhaltigkeitsregeln wie Grundsätze der Sparsamkeit und Effizienz unter Anwendung in allen Dimensionen menschlicher Aktivitäten erfüllen. Im Hinblick auf das Alltagsleben bedeutet dies einen möglichst sparsamen, effizienten und wenig verschwenderischen Umgang nicht nur mit Stoff und Energie, sondern auch mit Raum und Zeit. Neben anderen Maßnahmen sollten 
solche Aktivitäten im Alltag zur Erreichung der Nachhaltigkeitsziele, darunter der Millenniumsziele, die Menschheit sich im Rahmen der UNO-Programme setzte, beitragen. Ihre Realisierung sollte gemäß der angenommenen Agenda erfolgen.

\section{EINE KONZEPTUALISIERUNG DES IMPERATIVS EINER NACHHALTIGKEIT IM ALLTAG}

Die axiomatische Feststellung Deine tägliche, einzelne Entscheidung hat Einfluss auf die Umwelt ist Grundlage, die den Imperativ einer Nachhaltigkeit im Alltag bedingt. Allerdings würde eine eindeutige Bezugnahme auf diese Herausforderung, die jeweils detaillierte Analysen der Auswirkungen jeder meiner zukünftigen Entscheidung erforderlich macht, angesichts einer Unmenge von Entscheidungen, die wir mehr oder weniger reflektiert im Alltag treffen, zu einem Impotenz-Syndrom oder zumindest zu einer traumatischen Erfahrung führen. Im Alltagsleben alle relevanten ökologischen, sozialen und wirtschaftlichen Folgen jeder meiner kleinen Entscheidung zu analysieren, ist gar nicht möglich.

Mindestens drei Gründe sind hierfür ausschlaggebend. Der erste hängt mit den selbstverständlichen Begrenzungen unserer persönlichen Möglichkeiten, die Folgen einer konkreten Entscheidung zu erkennen, zusammen. Solches Verfahren stellt einen komplexen Mechanismus dar, denn es bedarf einer detaillierten Analyse einer - räumlich wie zeitlich - umfangreichen Ursache-Wirkungs-Kette. Dabei sind nur wenige von uns im Besitz des entsprechenden Fachwissens aus unterschiedlichen Wissenschaftsbereichen wie Biologie, Ökologie, Medizin, Hydrogeologie etc. Damit haben wir aber keine realen Möglichkeiten, die Folgen unserer Entscheidungen zu erkennen und zu beurteilen. Der zweite Grund ist rein pragmatisch und mit dem Sinn der alltäglichen Entscheidungen verbunden. Auch wenn wir über erforderliches Wissen verfügen würden und bei jeder täglichen Entscheidung entsprechende Folgenanalysen und Folgenbeurteilungen durchführen könnten, würden das Wesen und der pragmatische Sinn dieser kleinen täglichen Akte verloren gehen. Statt dieser kleinen täglichen Entscheidungen würden aufwendige Beurteilungsverfahren unseren Alltag dominieren. Am wichtigsten ist jedoch der dritte Grund, der die weiteren Überlegungen determinieren wird. Die Alltagskultur ist gewohnheitsmäßig. Sie ist Resultat einer Routine und eingeübter Handlungsweisen. Die Determinanten des Alltags sind gewohnheitsbedingte Handlungsmuster. Entscheidungen, die den Alltag konstituieren, sind wiederholbar und wiederholend, tagtäglich sind es (fast) die gleichen Akte, die sich aus bestimmten allgemeinen Lebensorientierungen ergeben. Somit sind diese Handlungsweisen, dieses sich täglich wiederholende Treffen von zahlreichen Entscheidungen, die den Entscheidungen aus dem Vortag jeweils gleich bis fast gleich sind, von Interesse. Mit anderen Worten: Das Schema des Alltagshandelns eines Menschen, das sich in Routinen und Gewohnheiten manifestiert, wird zu einem für den Imperativ einer Nachhaltigkeit im Alltag zentralen, axialen und konstitutiven Element. Das eigentliche Anliegen des Konzeptes ist, diese Schemata und die ihnen zugrunde liegenden Routinen und Gewohnheiten in Richtung mehr Nachhaltigkeit zu verändern.

Trotz fortschreitender Variabilität und Wandelbarkeit der Kultur sind die meisten ihrer Erzeugnisse durch relative Beständigkeit gekennzeichnet. Einige von ihnen sind kanonische Bestandteile einer Kultur und sind damit relativ dauerhaft und beständig, andere wiederum haben einen peripheren Charakter, veralten schneller und sind damit 
weniger resistent gegen Kulturwande ${ }^{16}$. Die Normen und Werte, die dem Nachhaltigkeitswandel in der Kultur zugrunde liegen, scheinen kanonische Bestandteile der Kultur zu sein. Für eine langfristige Stabilisierung dieser Elemente ist allerdings ein umfassender Bildungsprozess erforderlich, der die Grundgedanken der Nachhaltigkeit im Bewusstsein der Menschen prägen soll. Bevor dies passiert, ist jedoch eine fortschreitende Inkulturation der Nachhaltigkeit, d.h. eine Inkorporierung der entsprechenden Visionen und Vorstellungen in die Alltagskultur des Einzelnen und der Gemeinschaften erforderlich. Diese Inhalte müssen von Menschen angeeignet werden. Einer der wichtigsten Wege zur Diffusion und Prägung dieses kanonischen Bestandteils der Kultur ist einfach der umfangreiche Gebrauch von vorhandenen und leicht zugänglichen Ratgebern, in denen die im Sinne der Nachhaltigkeit richtigen Weisen der täglichen Lebensführung und Alltagshandelns dargestellt werden ${ }^{17}$.

Von Kerngedanken der nachhaltigen Entwicklung können die folgenden, den Imperativ einer Nachhaltigkeit im Alltag mitbestimmenden Grundsätze abgeleitet werden:

- Sei ethisch und moralisch in deinem Alltagsverhalten und -handeln.

- Beurteile deine täglichen Gewohnheiten periodisch auf Nachhaltigkeit hin und verändere sie, wenn sie sich als nicht nachhaltig erweisen.

- Vermehre dein Wissenskapital und verwende die einschlägigen Ratgeber für eine nachhaltige(re) Lebensführung dazu.

- Sei effektiv und sparsam in deinem täglichen Gebrauch von Umweltgütern wie Wasser, Gas, Strom und Kraftstoffe.

- Gehe mit Nahrungsmitteln und Trinkwasser/Getränken nicht verschwenderisch um.

- Verwende Mehrzweckprodukte, Mehrwegflaschen, -einkaufstaschen und sonstige Mehrwegverpackungen statt Einweg- und Mehrfachverpackungen.

Die im Bereich der Alltagskultur im Hinblick auf Nachhaltigkeit durchgeführten Forschungsarbeiten ergeben, dass Verhaltensweisen im Umgang mit Medien wie Wasser, Wärme und Strom, aber auch Abfalltrennen und tägliche Mobilität außerhalb des Haushalts von entscheidender Bedeutung sind. So wurden die Verhaltensweisen in der Nutzung von Wasser und Energie, in der Abfallentsorgung und im Bereich von Transport und Mobilität zu den für die Auswirkungen unseres Alltagshandelns auf die Umwelt entscheidenden und determinierenden Faktoren erklärt ${ }^{18}$. Somit stellen diese die Gegenstandsdimension des Imperativs einer Nachhaltigkeit im Alltag dar.

${ }^{16}$ Vgl. J. Kozielecki, Transgresja i kultura [Transgression und Kultur]. Wydawnictwo Akademickie ŻAK, Warszawa 2002 (poln.).

${ }^{17}$ DEFRA (Department for Environment, Food and Rural Affairs) (Hrsg.), A Framework for ProEnvironmental Behaviours, London 2008; G. Seyfang, Shopping for Sustainability: Can Sustainable Consumption Promote Ecological Citizenship? In: Environmental Politics, 14 (2) 2005, S. 290-306.; M. Skowrońska, Komfort, czyli co oznacza mieszkać dobrze [Komfort - was heißt gut wohnen?], in: Kultura i Społeczeństwo, Bd. LIV (1) 2010, S. 87-109 (poln.); A. Tuker, A. Cohen, K. Hubacek, O. Mont, The Impacts of Household Consumption and Options for Change, in: Journal of Industrial Ecology, 14 (1) 2010, S. 13-30.

${ }^{18}$ M. Quitzau, I. Røpke, Bathroom Transformation From Hygiene To Well-Being? in: Home Cultures, 6 (3) 2009, S. 219-242; J. Laskowska-Otwińska, Podróże do pracy jako element nowego stylu życia. Postulaty ruchu społecznego Slow Down [Pendeln zur Arbeit als Element eines neuen Lebensstils. Forderungen der Slow-Down-Bewegung], in: Kultura i Społeczeństwo [Kultur und 


\section{6. ÄNDERUNG DER TÄGLICHEN HANDLUNGSMATRIX}

„Die Zukunft der Sauberkeit ist ein Rätsel, das - wie immer - von materiellen Ressourcen einer bestimmten Gesellschaft und der Evolution ihrer Mentalität abhängt. Beispielsweise würde nichts anderes unsere Wasch- und Badegewohnheiten schneller und gründlicher verändern als ernsthafter Mangel an Wasser“"19.

Schon seit vielen Jahren befinden wir uns auf dem Weg in eine nachhaltige Gesellschaft und Wirtschaft. Das ist die Folge einer geistigen Ernüchterung, die Anfang der 1980er Jahre zustande kam und aus der Einsicht erwuchs, dass es so nicht weitergehen kann. Seit jener Zeit geht die Suche nach entsprechenden Handlungs- und Verhaltensregeln voran, die das Schrumpfen von natürlichen und menschlich-sozialen Grundlagen der Wirtschaftsentwicklung verhindern. Das vorgeschlagene Konzept des Imperativs einer Nachhaltigkeit im Alltag knüpft an diese Strömung an und erwächst aus der Wahrnehmung der Mitverantwortung für die weitere Entwicklung.

Obwohl die formulierten Grundsätze des Imperativs einer Nachhaltigkeit im Alltag als einfach und leicht handhabbar zu sein erscheinen, wird ihre Umsetzung oder, präziser ausgedruckt, ihre Einprägung in die Matrix menschlichen Verhaltens im Alltag ein langfristiger Lernprozess sein, der multidimensionale Aktivitäten erfordern wird. Beginnen muss er in der Familie, die primär die persönliche Alltagskultur prägt. Erforderlich wäre hierzu auch eine tiefgreifende Veränderung oder gar ein Umbruch im System der Allgemeinbildung, die auf Herausbildung des Systemverständnisses über die uns umgebende Wirklichkeit und auf die Wahrnehmung der Zusammenhänge und Kausalbeziehungen zwischen menschlichem Handeln und dem natürlichen und sozialen Umfeld abzielen, die eine Grundlage für die Verantwortungsübernahme für die (auch räumlich wie zeitlich fern liegenden) Folgen liefert. Herausgebildet werden müssen neben kognitiven Kompetenzen wie Fähigkeit zur Wahrnehmung von antagonistischen, synergetischen und additiven Phänomenen auch normative, moralische Fähigkeiten zum verantwortungsvollen Handeln. Darüber hinaus sollte durch Stärkung von den zum Kern der Nachhaltigkeit gehörenden geisteswissenschaftlichen und ökologischen Inhalten im Bildungssystem vermieden werden, dass sich technokratische Grundhaltungen und unbeschränktes Konsumverhalten weiter verbreiten.

\section{LITERATUR:}

[1] Appadurai A., Nowoczesność bez granic. Kulturowe wymiary globalizacji [Moderne ohne Grenzen. Kulturelle Dimensionen der Globalisierung], Univeritas, Kraków 2005 (poln.)

[2] Ashenburg K., Historia brudu [Die Geschichte des Schmutzes], Bellona S.A., Warszawa 2007 (poln.)

Gesellschaft]. Bd. LIV (1) 2010, S. 141-159 (poln.); J. Middleton, Sense and the city: exploring the embodied geographies of urban walking. In: Social \& Cultural Geography, 11(6) 2010, S. 575-596; OECD (Organisation for Economic Cooperation and Development) (Hrsg.), Towards Sustainable Household Consumption? Trends and Policies in OECD Countries, Paris 2002; OECD (Hrsg.), Promoting Sustainable Consumption, Good Practices in OECD Countries, Paris 2008.

${ }^{19}$ K. Ashenburg, Historia brudu [Geschichte des Schmutzes], op. cit., S. 248. 
[3] Baldwin E., Longhurst B., McCracken S., Ogborn M., Smith G., Wstęp do kulturoznawstwa [Einführung in die Kulturwissenschaft], Zysk i S-ka Wydawnictwo, Poznań 2007 (poln.)

[4] DEFRA, A Framework for Pro-Environmental Behaviours, Department for Environment, Food and Rural Affairs, London 2008.

[5] Gamble D. J., Destruction by insignificant increments. In: Northern Perspectives 7, 1979.

[6] Gram-Hanssen K., Consuming technologies - developing routines. In: Journal of Cleaner Production, 16(11) 2008.

[7] Gram-Hanssen K., Standby Consumption in Households Analyzed With a Practice Theory Approach. In: Journal of Industrial Ecology, 14(1), 2010.

[8] Harrison, L. E., Huntington, S. P. (Hrsg.), Kultura ma znaczenie [Kultur hat Bedeutung], Zysk i S-ka, Poznań 2003 (poln.)

[9] Head L., Muir P., Changing cultures of water in eastern Australian backyard gardens. In: Social \& Cultural Geography, 8(6), 2007.

[10]Janikowski R., Zarządzanie ekologiczne [Das Öko-Management]. Akademicka Oficyna Wydawnicza PLJ, Warszawa 1999 (poln.)

[11] Janikowski R., Kultura osia zrównoważonego rozwoju [Kultur als Achse nachhaltiger Entwicklung]. In: R. Janikowski, K. Krzysztofek (Hrsg.), Kultura a zrównoważony rozwój. Środowisko, ład przestrzenny, dziedzictwo [Kultur und nachhaltige Entwicklung. Umwelt, Raumordnung, Erbe], Polski Komitet do spraw UNESCO, Warszawa 2009 (poln.)

[12]Kozielecki J., Transgresja $i$ kultura [Transgression und Kultur]. Wydawnictwo Akademickie ŻAK, Warszawa 2002 (poln.)

[13] Laskowska-Otwińska J., Podróże do pracy jako element nowego stylu życia. Postulaty ruchu społecznego Slow Down [Pendeln zur Arbeit als Element eines neuen Lebensstils. Forderungen der Slow-Down-Bewegung]. In: Kultura i Społeczeństwo[Kultur und Gesellschaft] , Bd. LIV (1), 2010 (poln.)

[14] Meier A., Standby: Where are we now? In: Proceedings of ECEEE 2005 summer study: What works and who delivers? Paris: European Council for Energy Efficient Economy 2005.

[15]Michalski K., Interdyscyplinarność - transdyscyplinarność multidyscyplinarność nowy paradygmat $w$ nauce $i$ badaniach [Interdisziplinarität - Transdisziplinarität - Multidisziplinarität. Ein neues Paradigma in der Wissenschaft und Forschung]. In: Zeszyty Naukowe Politechniki Rzeszowskiej. Ekonomia $i$ Nauki Humanistyczne [Wissenschaftliche Hefte der Technischen Hochschule Rzeszow. Ökonomie und Geisteswissenschaften], Bd. 235, Heft 16, 2007 (poln.)

[16] Middleton J., Sense and the city: exploring the embodied geographies of urban walking. In: Social \& Cultural Geography, 11(6), 2010.

[17] Odum W. E., Environmental degradation and the tyranny of small decisions. In: Bioscience 32, 1982.

[18] OECD, Towards Sustainable Household Consumption? Trends and Policies in OECD Countries, Organisation for Economic Co-operation and Development, Paris 2002. 
[19] OECD, Promoting Sustainable Consumption, Good Practices in OECD Countries, Organisation for Economic Co-operation and Development, Paris 2008.

[20] Pennybacker M., Vibbert C., Do One Green Thing: Saving the Earth Through Simple, Everyday Choices, St. Martin's Griffin, New York 2010.

[21]Ponting C., A Green History of the World, Sinclair-Stevenson Ltd, London 1991.

[22] Quitzau M., Røpke I., Bathroom Transformation From Hygiene To WellBeing? In: Home Cultures, 6(3), 2009.

[23] Seyfang G., Shopping for Sustainability: Can Sustainable Consumption Promote Ecological Citizenship? In: Environmental Politics, 14(2), 2005.

[24] Skowrońska M., Komfort, czyli co oznacza mieszkać dobrze [Komfort - was heisst gut wohnen?]. In: Kultura i Społeczeństwo [Kultur und Gesellschaft]. Bd. LIV (1), 2010 (poln.)

[25] Toussaint-Samat M., Historia naturalna $i$ moralna jedzenia [Natur- und Moralgeschichte der Ernährung], Wydawnictwo W.A.B., Warszawa 2002 (poln.)

[26] Tuker A., Cohen A., Hubacek K., Mont O., The Impacts of Household Consumption and Options for Change. In: Journal of Industrial Ecology, 14 (1), 2010

\section{IMPERATYW ZRÓWNOWAŻONEJ CODZIENNOŚCI}

$\mathrm{Na}$ drodze do sustensywnego rozwoju jesteśmy już od wielu lat. Spowodowało to otrzeźwienie umysłowe jakie nastąpiło na początku lat osiemdziesiątych dwudziestego wieku wskazujące, że dalej tak być nie może. Od tego też czasu toczą się poszukiwania odpowiednich reguł postępowania. Także proponowany imperatyw włącza się do tego nurtu i współodpowiedzialności za dalszy rozwój. Koncepcja sustensywnego rozwoju opiera się na nałożeniu odpowiednich warunków. Dotyczy to także kultury codzienności. Kumulujące się oddziaływanie wielu indywidualnych działań na środowisko powoduje spotęgowany skutek wynikający z mnożnikowego i zagregowanego efektu indywidualnych decyzji. W naukach środowiskowych określa się to jako „destrukcja przez nieznaczne zmiany” lub „tyrania małych decyzji”. Aksjomatyczne stwierdzenie twoja codzienna indywidualna, jednostkowa decyzja ma wptyw na środowisko to podstawa warunkująca imperatyw zrównoważonej codzienności. Istotę imperatywu zrównoważonej codzienności tworzą zasady: bądź etyczny i moralny w swoim codziennym zachowaniu i działaniu; okresowo dokonuj oceny swoich codziennych nawyków, i zmieniaj je, jeśli nie są sustensywne; pomnażaj kapitał swojej wiedzy i korzystaj z poradników poprawnego sustensywnego działania; bądź efektywny i oszczędny w codziennym wykorzystywaniu mediów; nie bądź marnotrawny w odniesieniu do żywności i wody; korzystaj wielokrotnie z siatek, torebek i innych opakowań na zakupy. Bez wdrożenia w skali powszechnej zasad imperatywu zrównoważonej codzienności nie będzie możliwe zbliżanie się do sedna i istoty zrównoważonego rozwoju.

Słowa kluczowe: sustensywność, codzienność, zrównoważone decyzje

\section{IMPERATIVE OF SUSTAINABLE EVERYDAY}

For more than decade we have been following the "path" of sustainability. The sentence "it should not be like this anymore" has been a mental awakening for scantiest, academics, governments, non-governmental organizations and civil society. Since early seventies, we have been looking for relevant and sustainable rules of procedure, which are mainly based 
on intergenerational justice perceived as responsibility for future development. Concept of sustainable development includes imposition of appropriate conditions, that also applies to the "culture of everyday life". Cumulative impact of a multitude of individual actions on the environment causes heightened due to the multiplier effect and the aggregate effect of individual decisions. In the environmental sciences is referred to as the "destruction by insignificant increments" or "the tyranny of small decisions". Axiomatic statement: your daily individual, unitary decision has an impact on the environment is the basis for conditioning the imperative of sustainable everyday life. The essence of the imperative of sustainable everyday make the rules: be ethical and moral in their daily behavior and performance; perform periodic evaluations of their daily habits, and change them if they are not sustainable, multiply share their knowledge and use of proper instructions sustainable action; either efficient and cost-effective in the everyday use of the media; do not be wasteful in terms of food and water; using multiple bags and other packaging for shopping.

Keywords: sustainability, everyday life, sustainable decision.

DOI:10.7862/rz.2014.hss.37

Przesłano do redakcji: marzec 2014

Przyjęto do druku: październik 2014 